\title{
Assessment of xenoestrogenic exposure by a biomarker approach: application of the E-Screen bioassay to determine estrogenic response of serum extracts
}

\author{
Thomas Høj Rasmussen*1, Flemming Nielsen ${ }^{1}$, Helle Raun Andersen ${ }^{1}$, \\ Jesper Bo Nielsen ${ }^{1}$, Pal Weihe ${ }^{1,2}$ and Philippe Grandjean ${ }^{1,3}$
}

\begin{abstract}
Address: ${ }^{1}$ Department of Environmental Medicine, Institute of Public Health, University of Southern Denmark, Odense, Denmark, ${ }^{2}$ Department of Occupational and Public Health, Faroese Hospital System, Tórshavn, Faroe Islands and ${ }^{3}$ Department of Environmental Health, Harvard School of Public Health, Boston, MA 02115, USA
\end{abstract}

Email: Thomas Høj Rasmussen* - thoj@health.sdu.dk; Flemming Nielsen - fnielsen@health.sdu.dk;

Helle Raun Andersen - hrandersen@ health.sdu.dk; Jesper Bo Nielsen - jbnielsen@health.sdu.dk; Pal Weihe - pal@dfaa.hospital.fo;

Philippe Grandjean - pgrandjean@health.sdu.dk

* Corresponding author

Published: 15 October 2003

Environmental Health: A Global Access Science Source 2003, 2:12

This article is available from: http://www.ehjournal.net/content/2/1//2

(c) 2003 Rasmussen et al; licensee BioMed Central Ltd. This is an Open Access article: verbatim copying and redistribution of this article are permitted in all media for any purpose, provided this notice is preserved along with the article's original URL.
Received: 25 April 2003

Accepted: 15 October 2003

\begin{abstract}
Background: Epidemiological documentation of endocrine disruption is complicated by imprecise exposure assessment, especially when exposures are mixed. Even if the estrogenic activity of all compounds were known, the combined effect of possible additive and/or inhibiting interaction of xenoestrogens in a biological sample may be difficult to predict from chemical analysis of single compounds alone. Thus, analysis of mixtures allows evaluation of combined effects of chemicals each present at low concentrations.
\end{abstract}

Methods: We have developed an optimized in vitro E-Screen test to assess the combined functional estrogenic response of human serum. The xenoestrogens in serum were separated from endogenous steroids and pharmaceuticals by solid-phase extraction followed by fractionation by high-performance liquid chromatography. After dissolution of the isolated fraction in ethanolDMSO, the reconstituted extract was added with estrogen-depleted fetal calf serum to MCF-7 cells, the growth of which is stimulated by estrogen. After a 6-day incubation on a microwell plate, cell proliferation was assessed and compared with the effect of a 17-beta-estradiol standard.

Results and conclusions: To determine the applicability of this approach, we assessed the estrogenicity of serum samples from 30 pregnant and 60 non-pregnant Danish women thought to be exposed only to low levels of endocrine disruptors. We also studied $2 \mathrm{II}$ serum samples from pregnant Faroese women, whose marine diet included whale blubber that contain a high concentration of persistent halogenated pollutants. The estrogenicity of the serum from Danish controls exceeded the background in $22.7 \%$ of the cases, while the same was true for $68.1 \%$ of the Faroese samples. The increased estrogenicity response did not correlate with the lipid-based concentrations of individual suspected endocrine disruptors in the Faroese samples. When added along with the estradiol standard, an indication of an enhanced estrogenic response was found in most cases. Thus, the in vitro estrogenicity response offers a promising and feasible approach for an aggregated exposure assessment for xenoestrogens in serum. 


\section{Background}

Epidemiological research on endocrine disruption is hampered by difficulties in assessment of mixed exposures. A limited number of environmental chemicals have been characterized with regard to hormonal effects, but the effect of complex mixtures is virtually unknown. A promising approach to assess the combined functional estrogenic response in a serum sample was published by Sonnenschein, Soto and coworkers in 1995 [1]. This approach has been further developed and refined by research groups in the US [2], in Denmark [3] and in Spain [4].

Although logical and highly attractive, methodological difficulties must be overcome. Endogenous and pharmaceutical estrogens must be removed to avoid interference with effects caused by environmental chemicals. However, any pretreatment procedure must at the same time preserve the environmental chemicals of interest. Due to similar characteristics of the exogenous compounds that may bind to the estrogen receptor, this conflict is unlikely to be resolved in an ideal way. Pretreatment procedures are limited to physicochemical procedures that will not necessarily reflect the origin of the compounds and their physiological properties. Fortunately, many xenoestrogens identified so far are generally lipophilic, thus suggesting that a separation based on lipophilicity may constitute a feasible compromise.

We have refined and modified the original biomarker method [1] by $i$ ) introducing solid-phase extraction (SPE) of the serum sample with a newly developed SPE-polymeric sorbent that exhibits both hydrophilic and lipophilic retention characteristics [5], thereby ensuring extraction of a wider range of compounds according to polarity, ii) using a modified high-performance liquid chromatography (HPLC) gradient that covers the midpolar area for collection of the early lipophilic fraction before elution of endogenous estrogens, oral contraceptives and metabolites, and iii) applying a modified EScreen bioassay [6] that requires smaller amounts of serum extract and exhibits an increased ability to detect low-potency estrogenic compounds.

This methodology was applied on a substantial number of endogenous hormones, oral contraceptives, and a wide range of environmental pollutants known to be present in human serum. The information on specific retention times was used to optimize the HPLC separation procedure to include major xenoestrogens, while excluding interfering substances.

The biomarker method was used to assess the xenoestrogenic response of serum samples from 211 pregnant Faroese women ( $34^{\text {th }}$ week). This group had a high level of endogenous estrogens due to pregnancy, and, at the same time, a complex, mixed dietary exposure to xenoestrogens, including PCBs and other persistent halogenated pollutants that mainly originate from pilot whale blubber and fish $[7,8]$. A large number of halogenated organic pollutants are known to occur in biological matrices [9-13], but the quantitatively dominating environmental pollutants identified in human samples are the major $p, p^{\prime}$-DDT metabolite, $p, p^{\prime}-\mathrm{DDE}$, and the PCB congeners 2,2',3,4,4',5'-hexachlorobiphenyl (PCB138), 2,2',4,4',5, 5'-hexachlorobiphenyl (PCB153), and 2,2',3,4,4',5,5'heptachlorobiphenyl (PCB180), whereas the hydroxylated metabolites of PCBs are dominated by $4-\mathrm{OH}-$ $2,2^{\prime}, 3,4^{\prime}, 5,5^{\prime}, \quad$ 6-heptachlorobiphenyl (4-OH-CB187) $[8,9,12,14]$.

\section{Methods \\ Materials}

All media ingredients were purchased from In Vitro A/S (Fredensborg, Denmark) except insulin, sulforhodamine B (SRB), 2-amino-2-(hydroxymethyl)-1,3-propanediol (Tris base), trichloroacetic acid (TCA), dimethylsulfoxide (DMSO) Hybri-Max ${ }^{\circledast}(99.7 \%$ purity) that were purchased from Sigma (St. Louis, MO, US) and gentamicin sulfate (DuraScan, Odense, Denmark). The charcoal/dextrantreated fetal calf serum (CT-FCS) batch originated from the same lot (Lot No. 416632) as the untreated batch (Biological Industries, Beit Haemek, Israel), and was used in all experiments. The estrogen antagonist ICI 182,780 (ICI) was kindly provided by Astra Zeneca (Cheshire, UK). 17-beta-estradiol (E2), $o, p^{\prime}$-DDT, $p, p^{\prime}$-DDE, estrone, estriol, 16 $\alpha$-hydroxyestrone, progesterone, cholesterol, $5 \alpha$-dihydroprogesterone, $17 \alpha$-hydroxypregnenolone, androstenedione, dehydroepiandrosterone, 17 $\alpha$-hydroxyprogesterone were provided by Sigma (St. Louis, MO, US). Androsterone and 5 $\alpha$-dihydrotestosterone were obtained from Steraloids Inc (Newport, RI, US). Testosterone and ethanol $96 \%$ were obtained from Fluka (Buchs, Switzerland). $\beta-\mathrm{HCH}$ was provided by NSI Solutions Inc (Research Triangle Park, NC, US), methoxychlor, dieldrin, hexachlorobenzene (HCB), PCB105, PCB118, PCB138, PCB153, and PCB180 were provided from Dr. Ehrenstorfer GmbH (Augsburg, Germany). The hydroxylated PCBs, 4-OH-CB107, 4-OH-CB146 and 4-OH-CB187, were kindly provided by Dr. Aa. Bergman (Stockholm, Sweden). Methanol, $n$-heptane, ethyl acetate and isopropanol were of CHROMASOLV $^{\circledR}$ quality and obtained from Riedel-de Häen (Seelze, Germany), and 2,2,4-trimethylpentane was from Aldrich (Steinheim, Germany).

\section{Serum samples}

A cohort of 547 mother-child pairs was established consecutively at the National Hospital in Torshavn, Faroe Islands, and included women with a wide variation in exposure to persistent environmental pollutants due to 
differences in age and dietary habits. Maternal serum was collected at the last antenatal hospital visit at the $34^{\text {th }}$ week of pregnancy. Levels of individual exposures to PCBs and related compounds were characterized by analyses of milk collected from the same women 3-5 days after parturition; 211 women were then selected to represent the full range of exposures, with oversampling of subjects with low and high exposure levels.

As the women in this cohort had the same source of exposure (frequent marine food dinners, including pilot whale meat and blubber), the ratios between PCB congeners and related lipophilic pollutants would be expected to vary only little between the samples. The correlation coefficients between lipid-based concentrations of PCB105, PCB118, PCB138, PCB153, PCB156 or PCB180 and $o, p^{\prime}-$ DDT, $p, p$ '-DDE, HCB or trans-nonachlor were 0.90-0.95 (as also observed previously [15]), but milk lipid concentrations were used in this paper, as the higher fat content of milk would render these analyses more precise.

The cohort of Danish women consisted of 30 pregnant $\left(12^{\text {th }}-38^{\text {th }}\right.$ week) and 60 non-pregnant anonymous volunteers. Although we did not have exposure information for these women, the absence of whale blubber from the diet and the low-level exposures to persistent organohalogen compounds in Scandinavia suggest that they be considered controls for comparison with the Faroese cohort.

\section{Serum extraction method}

Solid-phase extraction was used to extract xenoestrogens from serum. Four mL serum was applied to an OASIS HLB 6 cc $500 \mathrm{mg}$ extraction cartridge (Waters, Milford, MA, US) placed in a Vac Elute SPS 24 Vacuum Manifold (Varian, Harbor City, CA, US). Each cartridge was conditioned with $3 \mathrm{~mL}$ methanol followed by $3 \mathrm{~mL}$ Milli-Q water prior to application of the serum. The serum was allowed to elute slowly (without applied vacuum) through the cartridges and dried with applied vacuum. The extracted compounds were eluted by $3 \mathrm{~mL}$ of methanol followed by $2 \mathrm{~mL}$ ethyl acetate into an extraction tube, and evaporated at $40^{\circ} \mathrm{C}$ under a gentle nitrogen stream. The remaining aqueous phase in the extraction tube was further extracted three times by $1 \mathrm{~mL} n$-heptane/ethyl acetate (1:1, vol/vol) and centrifuged at $1000 \mathrm{~g}$ in $10 \mathrm{~min}$. Following extraction, the three supernatants were pooled in a new centrifuge tube, evaporated and reconstituted in $125 \mu \mathrm{L} n$-heptane/ ethyl acetate (9:1, vol/vol). An aliquot of $100 \mu \mathrm{L}$ was injected into the HPLC system.

\section{Apparatus and chromatographic conditions (HPLC and GC)}

High performance liquid chromatography separation of the extracted compounds was performed using a LaChrom HPLC system consisting of a L-7100 HPLC pump, a L-7200 Auto Sampler, a L-7400 UV-detector, a L7450 Diode-array Detector, a L-7350 Column Thermostat and a D-7000 Interface Module (Merck, Darmstadt, Germany). Defined fractions of the mobile phase were collected by an L-7650 Fraction Collector (Merck, Darmstadt, Germany). The system was equipped with a LiChrospher Silica $60(5 \mu \mathrm{m}) 250 \times 4.6 \mathrm{~mm}$ column (Merck, Darmstadt, Germany). Elution of the compounds was performed using the gradient system: 0 - $18 \mathrm{~min}$ : $90 \%$ mobile phase A (100\% $n$-heptane) and $10 \%$ mobile phase B (methanol/n-heptane/ isopropanol (45:40:15, $\mathrm{vol} / \mathrm{vol} / \mathrm{vol})$ ); 18 - $22 \mathrm{~min}$ : gradient to $50 \% \mathrm{~A}$ and $50 \%$ B; 22 - 26 min: gradient to $100 \% \mathrm{~B} ; 26-28 \mathrm{~min}: 100 \%$ B; $28-33$ min: gradient to $90 \%$ A and $10 \%$ B. $33-35$ min: $90 \% \mathrm{~A}$ and $10 \% \mathrm{~B}$.

Gas chromatography (GC) was performed on a HewlettPackard 6890 GC equipped with a $\mu$-ECD and a 7683 series injector. Separation was performed on a HP-5MS capillary column $(30 \mathrm{~m} \times 0.25 \mathrm{~mm}, 0.25 \mu \mathrm{m}$ film thickness).

\section{Preparation of samples for E-Screen bioassay}

Prior to each analysis the retention time of a standard mixture, including $5 \alpha$-dihydroprogesterone, testosterone and estrone, was used to control and adjust time windows for collection of the HPLC fractions.

The fractions were collected in glass tubes and evaporated to dryness at $40^{\circ} \mathrm{C}$ under a gentle nitrogen stream. The dried sample was reconstituted in $20 \mu \mathrm{L} \mathrm{EtOH/DMSO/}$ Milli-Q water (50:10:40, vol/vol/vol) by vortexing for 30 sec. Then $200 \mu \mathrm{L}$ CT-FCS was added and again the content was mixed by vortexing. The sample was divided in two aliquots of $100 \mu \mathrm{L}$ in polypropylene tubes; tube A and $\mathrm{B}$. To tube A, $900 \mu \mathrm{L}$ estrogen-free medium was added. From this solution, aliquots of $200 \mu \mathrm{L}$ and $100 \mu \mathrm{L}$ were transferred to new polypropylene tubes containing $800 \mu \mathrm{L}$ and $900 \mu \mathrm{L}$ estrogen-free medium, respectively. To tube B, 900 $\mu \mathrm{L}$ estrogen-free medium supplemented with $10 \mathrm{pM}$ E2 was added, after which aliquots of $200 \mu \mathrm{L}$ and $100 \mu \mathrm{L}$ were transferred to new polypropylene tubes containing $800 \mu \mathrm{L}$ and $900 \mu \mathrm{L}$ estrogen-free medium supplemented with 10 pM E2, respectively. Thus, all serum samples were analyzed as undiluted samples and as 5- and 10-fold dilutions as well as with and without E2. Prior to the analysis, it was confirmed that the solvent concentration $(0.5 \%$ ethanol and $0.1 \%$ DMSO) in the final samples did not affect cell proliferation. Sterile filtering of the samples was unnecessary and the samples were prepared immediately prior to use.

\section{Recovery of xenoestrogens after reconstitution}

Stock solutions of PCB118, PCB153,, , $p^{\prime}$-DDT and $p, p^{\prime}$ $\mathrm{DDE}$, either alone or in mixture, were diluted to a final 
concentration of $50 \mathrm{nM}$ in $13 \mathrm{~mL}$ HPLC mobile phase (90 $\% \mathrm{~A}$ and $10 \% \mathrm{~B}$ ) in glass tubes. The sample was divided in two aliquots of $6.225 \mathrm{~mL}$ (equal to the volume of Fraction-1) in glass tubes; tube 1 and 2 . Tube 1 was evaporated to dryness at $40^{\circ} \mathrm{C}$ under a gentle nitrogen stream. The dried sample was reconstituted in $20 \mu \mathrm{L} \mathrm{EtOH/DMSO/}$ Milli-Q water (50:10:40, vol/vol/vol) by vortexing for 30 sec. Then, $200 \mu \mathrm{L}$ CT-FCS was added and again the content was mixed by vortexing. Finally, $1.8 \mathrm{~mL}$ estrogen-free medium was added to the vial, after which an aliquot of $2.0 \mathrm{~mL}$ was extracted as described for serum samples by Brock et al, 1996 [16] using a Strata $500 \mathrm{mg}$ trifunctional $\mathrm{C}_{18 \text {-e }}$ SPE column and a Strata $500 \mathrm{mg}$ Florisil Pesticide Residue column (Phenomenex, Torrance, CA). The eluted solvents were collected in a tarred disposable centrifugation tube and evaporated at $30^{\circ} \mathrm{C}$ under a gentle nitrogen stream to about $0.5 \mathrm{~mL}$. After weighing, the remaining amount was calculated and the volume adjusted to 1000 $\mu \mathrm{L}$ with 2,2,4-trimethylpentane. Tube 2 was evaporated at $40^{\circ} \mathrm{C}$ under a gentle nitrogen stream to about $0.25 \mathrm{~mL}$. Again, the remaining amount was calculated, and the volume adjusted to $1000 \mu \mathrm{L}$ with 2,2,4-trimethylpentane. The concentration was determined in an aliquot of $2 \mu \mathrm{L}$ by GC analysis.

\section{Microwell E-Screen bioassay}

Stock cultures of MCF-7 BUS cells (passage 113-120) were grown in Dulbecco's modified Eagle's medium (DMEM) containing $10 \%$ FCS supplemented with $4 \mathrm{mM}$ glutamine, $15 \mathrm{mM}$ 4-(2-hydroxyethyl)-1-piperazineethanesulfonic acid (HEPES) and $54 \mu \mathrm{g} / \mathrm{L}$ gentamicin sulfate in an atmosphere of $5 \% \mathrm{CO}_{2} / 95 \%$ air under saturating humidity at $37^{\circ} \mathrm{C}$. The microwell E-Screen was performed as described in Rasmussen and Nielsen, 2002 [6]. Briefly, cells (4500 cells/well) were seeded in 96-well Nunclon Delta MicroWell plates (Nunc, Roskilde, Denmark) and after $24 \mathrm{hr}$, samples were added in estrogenfree medium (phenol red-free DMEM containing 10\% CTFCS supplemented with $4 \mathrm{mM}$ glutamine, $20 \mathrm{mM}$ HEPES, $0.1 \%$ sodium bicarbonate and $3 \mu \mathrm{g} / \mathrm{L}$ insulin). In each experiment, a standard curve for $1 \mathrm{pM}-10 \mathrm{nM}$ E2 was included. After 6 days, cells were fixed with TCA and stained with SRB, after which bound dye was solubilized with $200 \mu \mathrm{L}$ Tris base. The absorbance at $492 \mathrm{~nm}$ was determined directly in the microwell culture plate using a microplate reader (Dynatech MRX, Chantilly, VA, US).

\section{Quantitative evaluation}

\section{Proliferative effect (PE)}

The endpoint of the E-Screen bioassay is the number of cells after treatment compared to the number of cells in a negative control sample (cell treated with estrogen-free medium supplemented with solvent only). Thus, the PE was calculated as the ratio between the maximum cell pro- liferation achieved with the test sample and with the negative control:

$$
\text { Equation } 1: \text { PE }=\frac{\text { cell number }(\text { test sample) }}{\text { cell number (negative control) }}
$$

Relative proliferative effect (RPE)

For each test sample the RPE was calculated as suggested by Sonnenschein, Soto and coworkers in 1995 [1] from the PE of the test sample and the PE of $1 \mathrm{nM}$ E2 in the same experiment:

Equation $2: \mathrm{RPE}=\left(\frac{\left(\mathrm{PE}_{\text {test sample }}-1\right)}{\left(\mathrm{PE}_{\mathrm{E} 2}-1\right)}\right) \times 100$

Because $1 \mathrm{nM}$ E2 induces maximum response in the EScreen bioassay, the RPE describes the ability of the test sample to induce cell proliferation relative to maximum E2-induced cell proliferation. Thus, the RPE describes the efficacy of the test sample relative to that of E2.

However, the PE of 10 pM E2 was used to calculate the RPE of those experiments where mixture effects were studied because this concentration induce one-half of the maximum response. Thus, this RPE describes whether the test sample has enhancing or inhibiting effects on E2induced cell proliferation, e.g., have RPE values above or below $100 \%$.

\section{Relative proliferative potency (RPP)}

For each individual compound, the RPP was calculated as suggested by Sonnenschein, Soto and coworkers in 1995 [1] as the ratio between the lowest concentration of E2 that induce maximum response $\left(\mathrm{MOEC}_{\mathrm{E} 2}\right.$, maximum observed effect concentration) and the lowest concentration of test compound that induce maximum response (MOEC test compound $_{\text {): }}$

Equation 3: RPP $=\left(\frac{\text { MOEC }_{\mathrm{E} 2}}{\mathrm{MOEC}_{\text {test compound }}}\right) \times 100$

Thus, the RPP describes the potency of the test compound relative to that of $\mathrm{E} 2$.

The estrogenic response of a serum sample was regarded positive when its RPE value was significantly above background noise, i.e., the variability of negative control values. For the biomarker assay, the background was defined as the mean of solvent-treated controls plus three times the standard deviation.

\section{Statistical analysis}

Concentration-response relationships for individual compounds were based on at least three independent 
experiments. Homogeneity of variance between the experiments was tested by Bartlett's test. The data were logarithmically transformed if necessary before analysis of variance (ANOVA). If the ANOVA test showed a significant difference $(\mathrm{P}<0.05)$, the Tukey's Multiple Comparisons Test was used to identify differences between means. Associations between the biomarker response determined in serum and the concentration of environmental pollutants determined in milk were calculated using Pearson correlation coefficients. The data were logarithmically transformed before conducting the analysis.

\section{Results}

\section{Establishment of HPLC fractions}

The advantage of using normal phase chromatography (NPC), where apolar substances are eluted prior to more polar substances, is obvious for many reasons. A range of identified endocrine disrupters are by nature more lipophilic than the endogenous hormones, and elute therefore prior to the range of hormones and their metabolites in a NPC system. Furthermore, this system utilizes water free eluents, thereby allowing evaporation of solvents at relative low temperatures and thus minimizing the risk of a loss of compounds with low vapor pressure. The use of a gradient system is essential to elute the most hydrophilic substances prior to the next injection.

Eighty-eight compounds (including alkyl phenols, phthalates, PCBs, organochlorine and carbamate pesticides, oral contraceptives, and endogenous estrogens) were used to characterize the HPLC separation (Appendix [see 1]). The compounds were monitored by diode-array detection, and a library based on retention time as well as the
UV-spectrum was generated. Calibration curves were produced for solutions of the compounds at several relevant concentrations. Recovery studies were performed by comparing the peak area obtained from a supplemented serum sample, extracted and analyzed as described above, with the peak area obtained from direct injection of an equivalent amount of the pure standard. The recoveries of selected compounds relevant for this cohort study are presented in Table 1 . The chromatographic conditions were developed in order to elute a wide range of xenoestrogens and other environmental pollutants within the early fractionation period, i.e., before a range of endogenous estrogens, oral contraceptives, and metabolites eluted. Based on the library of specific retention times combined with information on the estrogenic activity, a HPLC fraction (Fr-1) was defined that consisted of the initial $5.5 \mathrm{~min}$ of the HPLC eluate. This Fr-1 included the most dominant parent PCB congeners and some of the hydroxylated PCB metabolites in biological extracts, but excluded almost all endogenous estrogens and their metabolites (Table 2).

Table I: Recovery (\%) of supplemented organochlorines from CT-FCS after HPLC separation

\begin{tabular}{lc}
\hline Compound & Recovery \\
\hline o,p'-DDT & 89 \\
p,p'-DDE & 85 \\
Methoxychlor & 76 \\
PCB52 & 61 \\
PCBII8 & 55 \\
PCBI53 & 67 \\
PCBI80 & 54 \\
\hline
\end{tabular}

Table 2: Retention time and estrogenic response of indicative compounds

\begin{tabular}{|c|c|c|c|c|}
\hline \multirow[t]{2}{*}{ Compound } & \multirow[t]{2}{*}{ Rt } & \multicolumn{2}{|l|}{ MOEC } & \multirow[t]{2}{*}{$\mathbf{R P P}$} \\
\hline & & $\mu \mathbf{M}$ & RPE \pm SD & \\
\hline \multicolumn{5}{|l|}{ Endogenous compounds } \\
\hline Cholesterol & 1.83 & $0.0001-10$ & Nonactive & - \\
\hline $5 \alpha$-dihydroprogesterone & 4.14 & 10 & $58 \pm 3$ & 0.01 \\
\hline Progesterone & 5.03 & 25 & $16 \pm 2$ & 0.004 \\
\hline Pregnenolone & 6.07 & 10 & $92 \pm 6$ & 0.01 \\
\hline Androsterone & 6.21 & 10 & $97 \pm 3$ & 0.01 \\
\hline $5 \alpha$-dihydrotestosterone & 6.63 & 1 & $64 \pm 5$ & 0.1 \\
\hline Androstenedione & 6.80 & 10 & $16 \pm 2$ & 0.01 \\
\hline Dehydroepiandrosterone & 6.86 & 1 & $92 \pm 2$ & 0.1 \\
\hline Testosterone & 8.23 & 10 & $34 \pm 5$ & 0.01 \\
\hline Estrone & 9.06 & 0.01 & $84 \pm 8$ & 10 \\
\hline I7 $\alpha$-hydroxyprogesterone & 9.23 & 10 & $7 \pm 1$ & 0.01 \\
\hline I7 $\alpha$-hydroxypregnenolone & 12.78 & 10 & $30 \pm 1$ & 0.01 \\
\hline I7 $\beta$-estradiol & 14.83 & 0.001 & $100 \pm 7$ & 100 \\
\hline 16 $\alpha$-hydroxyestrone & 22.86 & 0.01 & $77 \pm 2$ & 10 \\
\hline Estriol & 24.01 & 0.01 & $103 \pm 3$ & 10 \\
\hline \multicolumn{5}{|l|}{ Organochlorines } \\
\hline Hexachlorobenzene & 1.34 & $0.001-50$ & Nonactive & - \\
\hline
\end{tabular}


Table 2: Retention time and estrogenic response of indicative compounds (Continued)

\begin{tabular}{|c|c|c|c|c|}
\hline PCBI80 & 1.40 & $0.001-50$ & Nonactive & - \\
\hline$p, p^{\prime}-\mathrm{DDE}$ & 1.43 & 12.5 & $92 \pm 2$ & 0.008 \\
\hline PCBI53 & 1.48 & $0.001-50$ & Nonactive & - \\
\hline PCBI38 & 1.49 & $0.001-50$ & Nonactive & - \\
\hline$o, p^{\prime}-\mathrm{DDT}$ & 1.53 & 6.25 & $96 \pm 7$ & 0.016 \\
\hline Dieldrin & 1.75 & 25 & $44 \pm 9$ & 0.004 \\
\hline Methoxychlor & $2.29+2.88$ & 12.5 & $57 \pm 8$ & 0.008 \\
\hline 4-OH-CBI 46 & 2.67 & $0.001-50$ & Nonactive & - \\
\hline $4-\mathrm{OH}-\mathrm{CB} / 87$ & 2.67 & 50 & $24 \pm 4$ & 0.002 \\
\hline 4-OH-CBIO7 & 2.70 & $0.001-50$ & Nonactive & - \\
\hline$\beta$-hexachlorocyclohexane & 4.13 & 2.5 & $61 \pm 2$ & 0.04 \\
\hline
\end{tabular}

Abbreviations: Rt, retention time; MOEC, maximum observed effect concentration; RPE, relative proliferative effect; RPP, relative proliferative potency

\section{Recovery of organochlorines after reconstitution}

To evaluate our protocol for reconstitution of dried HPLC fractions in cell culture medium, the recoveries of indicative organochlorines were determined. Stock solutions of PCB118, PCB153, $o, p^{\prime}$-DDT and $p, p^{\prime}$-DDE were diluted to $50 \mathrm{nM}$ in HPLC mobile phase. The samples were evaporated to dryness and reconstituted using our protocol described for reconstitution of serum samples, followed by determination of the concentration by GC analysis. The concentration was concomitantly determined in control samples, and, since the specific recoveries of the investigated compounds were known (data not shown), we were able to calculate the recovery of the xenoestrogens in the reconstituted samples. Specific recoveries in the reconstituted samples in cell culture medium are presented in Table 3. The experiments were repeated with a mixture of the four compounds, and equivalent percentages were recovered (Table 3 ).

Table 3: Comparison of the recovery (\%) of organochlorines (50 $\mathrm{nM}$ each) from reconstituted samples supplemented with a single compound or a mixture of four.

\begin{tabular}{|c|c|c|}
\hline \multirow[t]{2}{*}{ Compound } & \multicolumn{2}{|c|}{ Recovery } \\
\hline & Single & Mixture \\
\hline$o, p^{\prime}-\mathrm{DDT}$ & 54 & 61 \\
\hline$p, p^{\prime}-\mathrm{DDE}$ & 58 & 66 \\
\hline PCBII8 & 72 & 67 \\
\hline PCBI53 & 81 & 80 \\
\hline
\end{tabular}

\section{Functional estrogenic response of selected environmental pollutants}

The estrogenicity of major environmental pollutants previously identified in serum from the cohort of pregnant Faeroese women was determined. The organochlorine insecticide, $o, p$ '-DDT had efficacies equivalent to E2, giving a maximum RPE of $96 \pm 7$ (Figure 1). The more stable metabolite of $p, p^{\prime}$-DDT, $p, p^{\prime}$-DDE, also had a high efficacy, giving a maximum RPE of $92 \pm 2$ (Figure 1 ). The maximum response to $o, p^{\prime}$-DDT was observed upon treatment with $6.25 \mu \mathrm{M}$ whereas a concentration of $12.5 \mu \mathrm{M}$ was needed to produce an equivalent effect upon treatment with $p, p^{\prime}$-DDE. Thus, $o, p^{\prime}$-DDT had a higher potency (RPP $=0.016)$ than $p, p^{\prime}-\mathrm{DDE}(0.008)$ (Table 2$)$. When cells were cotreated with $10 \mathrm{pM}$ E2 (which induce one-half of the maximum response) and $o, p^{\prime}$-DDT or $p, p^{\prime}$-DDE, an enhanced induction of cell proliferation was observed when compared to that of treatment with 10 pM E2 alone, indicating additive mixture effects. Another organochlorine insecticide, methoxychlor, had a potency equivalent to $p, p$-DDE, but a significantly lower efficacy (RPE $=57 \pm$ 8) (Table 2 and Figure 1). Methoxychlor also enhanced the induction of cell proliferation in the presence of E2, again indicating an additive mixture effect. The organochlorine insecticide dieldrin $(\mathrm{RPE}=44 \pm 9)$ had an efficacy equivalent to methoxychlor, but a lower potency (0.004) (Table 2 and Figure 1). None of the three major PCB congeners, PCB138, PCB153 and PCB180, which constitute about half of the total PCB concentration in serum from Faroese women [7], induced an estrogenic response (Figure 1). Also, when tested as a mixture of equimolar concentrations of the three PCB congeners, no induction of cell proliferation was detected (Figure 1). Cotreatment experiments with E2 indicated that each of these PCB congeners elicits a weak anti-estrogenic effect, since the proliferative effect of treatment with 10 pM E2 was reduced when compared to that of treatment with 10 pM E2 alone (Figure 1). Cotreatment with a mixture of the three congeners and E2 resulted in a comparable inhibition of the E2-induced proliferation (Figure 1). Cytotoxicity tests confirmed that these PCB concentrations were nontoxic (data not shown). The three most abundant hydroxylated metabolites of PCB congeners, 4-OHCB107, 4-OH-CB146 and 4-OH-CB187, found in serum from Faroese women, were examined as well. At the highest concentration analyzed, 4-OH-CB187 increased cell 

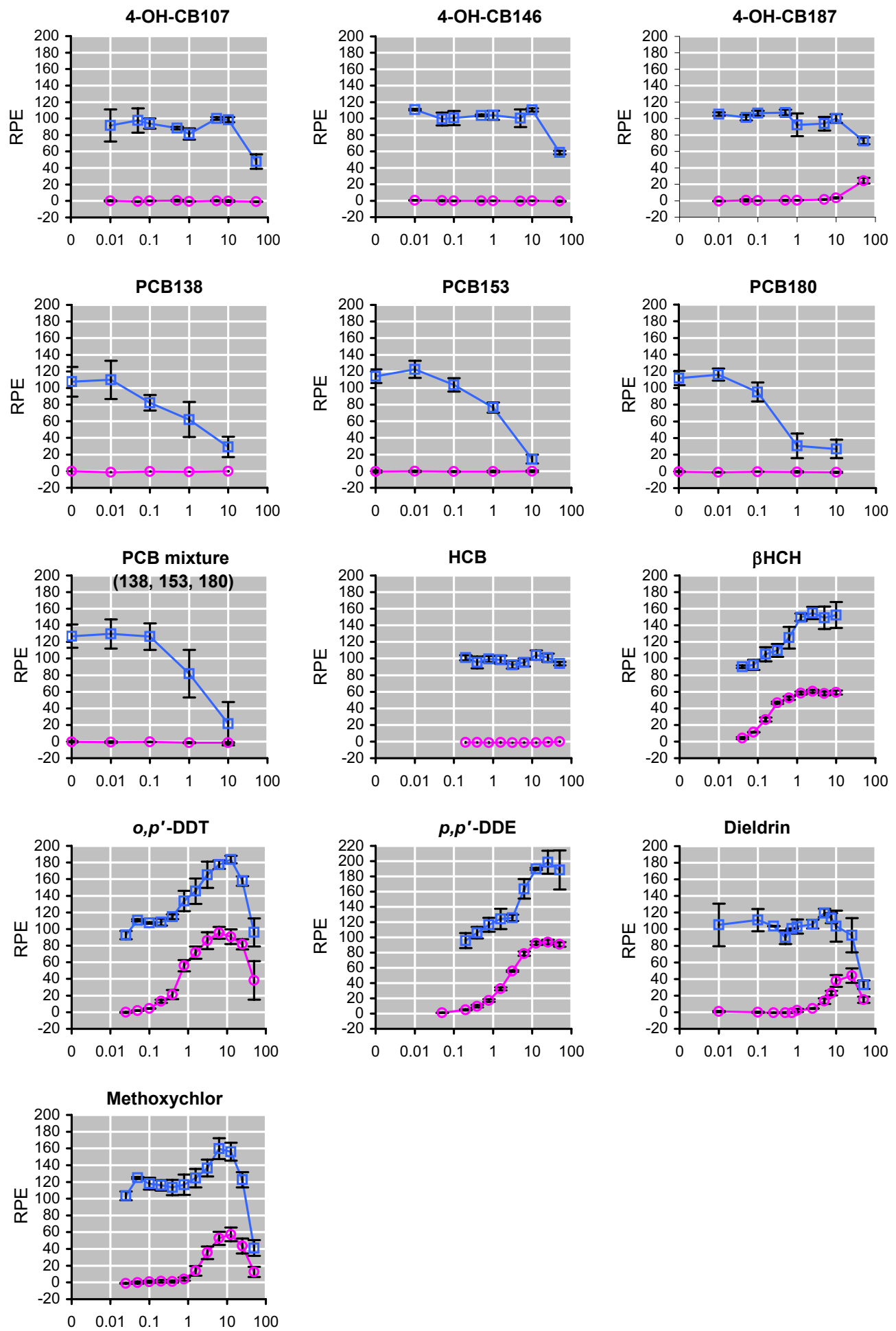

Figure I

E-Screen analysis of environmental pollutants. MCF-7 cells were treated with the compound indicated in the absence (O) or presence ( $\square$ ) of 10 pM E2. After seven days of culture, cell proliferation was determined using the SRB assay. Results are expressed as the relative proliferative effect (RPE) to E2 ( $y$-axis), and concentrations indicated are in $\mu M$ ( $x$-axis). Values shown are mean \pm SD from triplicates at two or more independent experiments. 
proliferation reaching a maximum RPE of $24 \pm 4$ whereas 4-OH-CB107 and 4-OH-CB146 did not induce cell proliferation in the concentration range tested (Figure 1 ). The prevalent environmental pollutant HCB, did not induce cell proliferation, nor affected the proliferative effect of 10 pM E2. In contrast, another environmental pollutant, $\beta$ $\mathrm{HCH}$, induced a marked concentration-dependent induction of cell proliferation, reaching maximum RPE of $61 \pm$ 2 upon treatment with a concentration of $2.5 \mu \mathrm{M}$. As observed for $o, p^{\prime}$-DDT, $p, p^{\prime}$-DDE and methoxychlor, $\beta$ $\mathrm{HCH}$ enhanced the proliferative effect in cotreatment with 10 pM E2, indicating an additive mixture effect.

These results therefore indicate that the parent PCB congeners that constitute the main part of the PCBs present in serum do not cause an estrogenic effect but rather an antiestrogenic effect, though other persistent pollutants usually occurring along with the PCBs may cause an estrogenic effect.

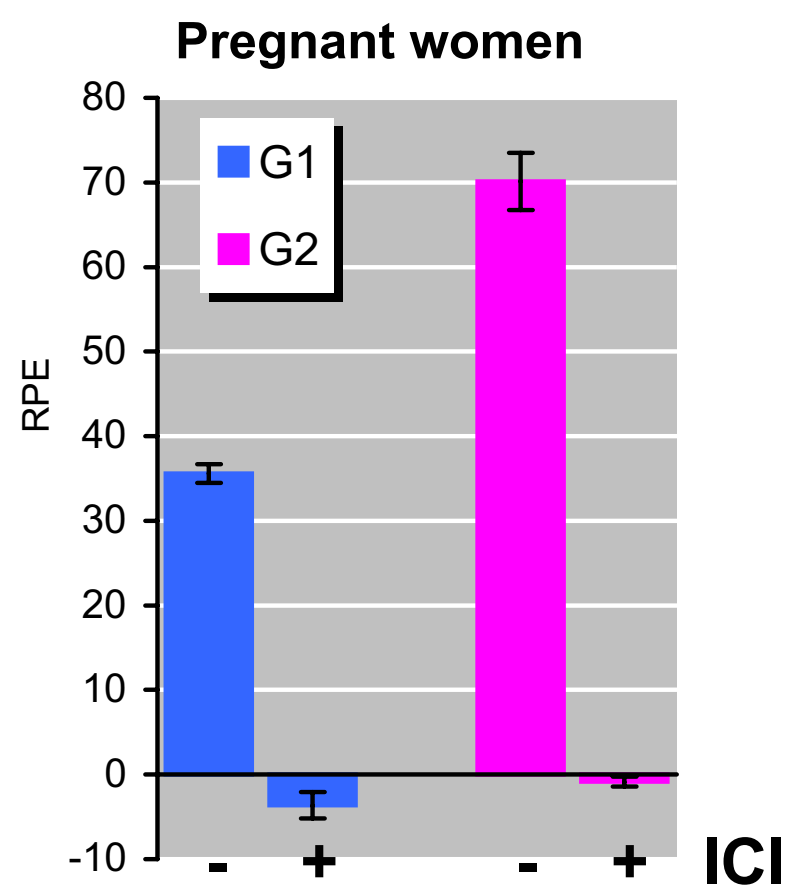

\section{Figure 3}

Biomarker analysis of Fraction-I from unexposed pregnant women. Duplicate serum samples from two unexposed pregnant women (GI and $\mathrm{G} 2$ ) from the control group were separated on HPLC, followed by E-Screen analysis of the reconstituted Fr-I (0-5.5 minutes). Fr-I was analyzed in the presence or absence of $\mathrm{ICl}$ as indicated. Results are expressed as the relative proliferative effect (RPE) to E2. Values shown are mean \pm SD from triplicate wells.

\section{Functional estrogenic response of serum from women}

Using the biomarker assay the combined functional estrogenic response of Fr-1 was determined from samples obtained from non-pregnant female volunteers from the Danish control group. Most samples did not induce a significant induction of cell proliferation (Figure 2, blue bars). However, a few samples did induce a modest cell proliferation, thus supporting the notion that this biomarker assay is very sensitive.

Detailed studies were then performed to examine the serum estrogenicity of Fr-1 of serum samples from two pregnant women (35th and $39^{\text {th }}$ week, respectively) from the Danish control group. Fr-1 from both pregnant women induced a marked cell proliferation reaching a maximum RPE of $73 \pm 4$ and $38 \pm 3$, respectively (Figure 3 ). Addition of $1 \mathrm{nM}$ ICI 182,780, a pure anti-estrogen that completely blocks estrogen receptor (ER)-mediated responses, completely inhibited cell proliferation induced by Fr-1 of both samples (Figure 3), thus demonstrating that the induced cell proliferation was mediated via ER. Subsequent analyses of subfractions of Fr- 1 indicated that the majority of the serum estrogenicity in both samples could be ascribed to two minor subfractions - namely s 4 (1.8-2.3 $\mathrm{min}$ ) and s8 (3.8-4.3 $\mathrm{min})$ (Figure 4). The resulting effect of the pooled subfractions indicated an additive mixture effect of the estrogenic substances present in subfraction $s 4$ and $s 8$. Subfractions $s 4$ and $s 8$ were then analyzed by MS and the endogenous steroid $5 \alpha$ dihydroprogesterone was identified in subfraction $\mathrm{s} 8$ (data not shown), and subsequent E-Screen analysis indicated that $5 \alpha$-dihydroprogesterone was a potent estrogenic compound with a maximum RPE of $58 \pm 3$ (Table 2). In subfraction s4, the only identifiable compound with a steroid structure was cholesterol, but subsequent E-Screen analysis indicated that cholesterol or cholesterol metabolites potentially formed by the MCF-7 cells during the bioassay did not induce cell proliferation. However, although fatty acid esters of estrone or estradiol may be present in serum [17], their potential occurrence in subfraction $\mathrm{s} 4$ could not be documented.

To avoid false positive results, both subfractions s 4 and s8 were removed to exclude possible pregnancy-related estrogens present in serum from pregnant women. The estrogenic response of the resulting Fr-1 extracts (Figure 2, purple bars) was not different from that of samples from non-pregnant women (Figure 2, blue bars) in this cohort, and the groups were therefore merged into one control cohort. Of this cohort, $22.7 \%$ of the samples induced cell proliferation above the background level. 


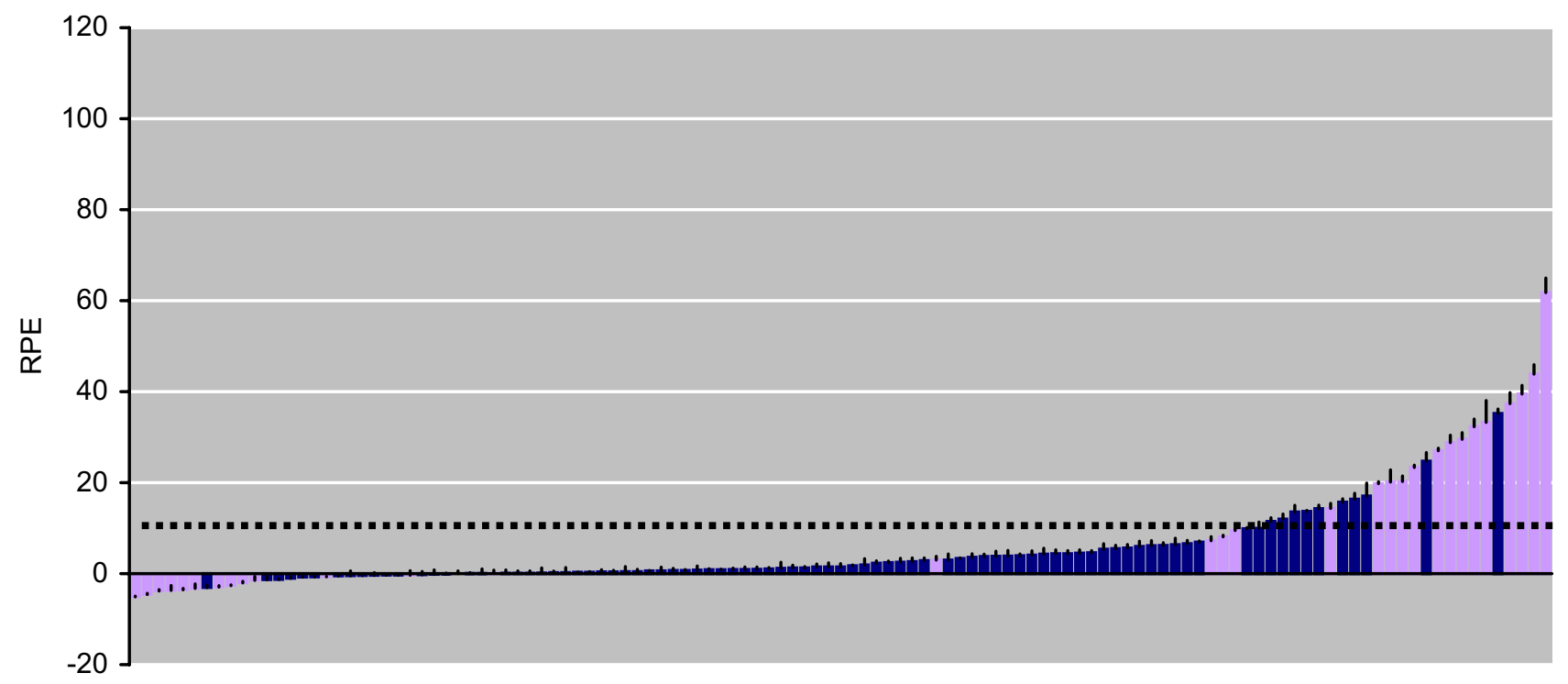

Samples $(\mathbf{n}=90)$

\section{Figure 2}

Biomarker analysis of Fraction-I from unexposed women. Serum samples from unexposed pregnant $(\mathrm{n}=30)$ (purple bars) and non-pregnant $(n=60)$ (blue bars) women were separated on HPLC, followed by E-Screen analysis of the reconstituted $\mathrm{Fr}-\mathrm{I}$ (0-5.5 minutes). For samples from pregnant women, subfractions s4 (I.8-2.3 minutes) and s8 (3.8-4.3 minutes) was removed before E-Screen analysis. Results are expressed as the relative proliferative effect (RPE) to E2. Values shown are mean \pm SD from triplicate wells. The broken line represents the mean background of the experimental series (the mean of negative controls $+3 x \mathrm{SD})$.

\section{Functional estrogenic response of serum from PCB- exposed women}

The serum estrogenicity was then determined from samples obtained from pregnant ( $34^{\text {th }}$ week) Faroese women. After HPLC separation, subfractions s4 and s8 were removed as described above, and all samples were tested separately by E-Screen analysis as undiluted and as 5- and 10 -fold dilutions; below we present the results from the undiluted samples.

As seen on Figure 5A, 68.1\% of the samples induced cell proliferation above the background level. Additionally, enhancing or inhibiting effects of Fr-1 were determined by co-treatment with 10 pM E2, which would induce onehalf of the maximum response in the E-Screen bioassay. A few $(9.0 \%)$ of the samples inhibited the effect of $10 \mathrm{pM}$ E2. All of these samples also exhibited reduced estrogenicity as undiluted samples without E2, as compared to 5fold dilutions without E2. Most of the remaining samples, when tested as undiluted samples, enhanced the effect of 10 pM E2 and the results from the unspiked extracts indicated that the mixture effect was additive (Figure 5B).

\section{Correlation between environmental pollutant concentration and biomarker response}

The concentration of environmental pollutants in breast milk from the subjects examined had been determined by GC analyses (Heinzow B. Unpublished data). The biomarker results were compared with the lipid-based concentrations of PCBs and related pollutants. However, except for $\beta-\mathrm{HCH}$, no statistically significant correlations were found between serum estrogenicity and any of the organochlorines analyzed (results not shown). The samples were then divided into two groups with high and low total PCB concentration, respectively, and the groups were compared with the pollutant levels individually. Again, no association with estrogenic response was observed. Correlation analyses between the serum antiestrogenic results and the lipid-based concentrations of PCBs and related pollutants were also performed but no statistically significant correlations were found between the biomarker response and any of the organochlorines analyzed.

\section{Discussion \\ Estrogenicity of environmental pollutants}

The estrogenic activities of a range of individual compounds in this study confirm the reported estrogenicity of 


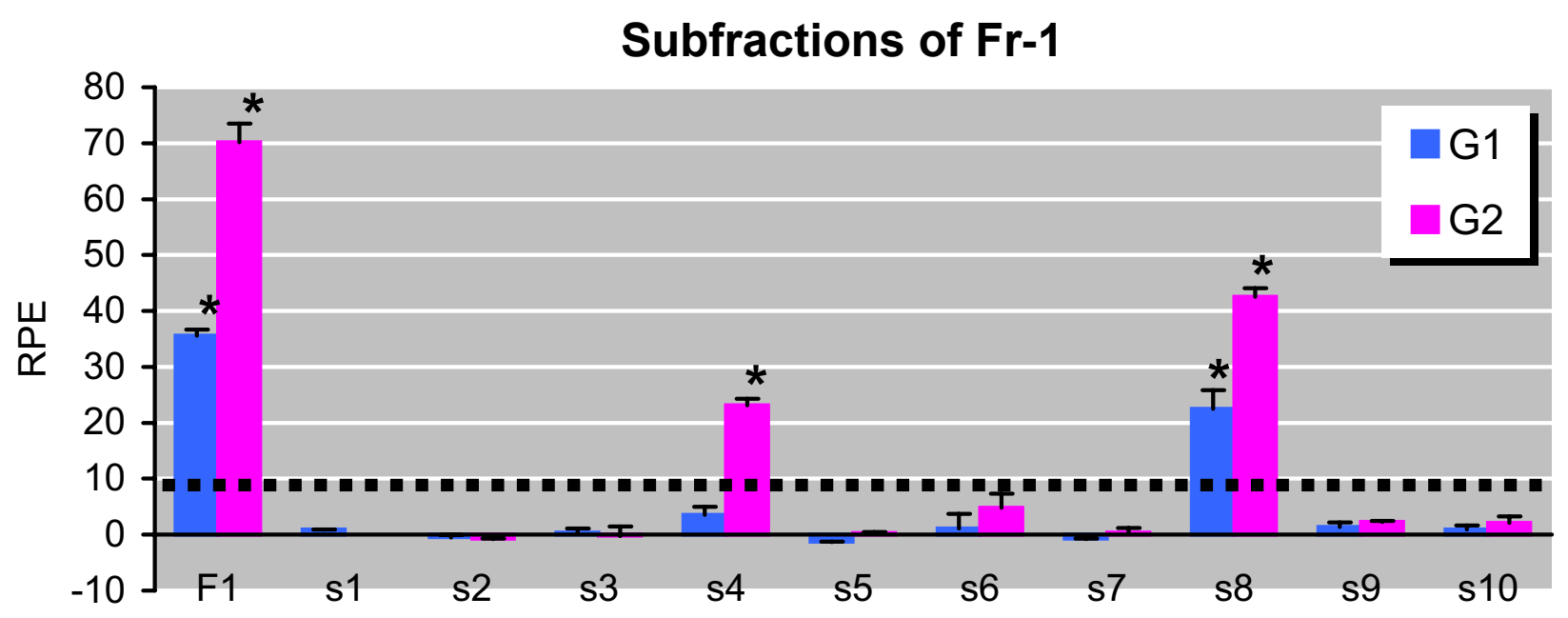

\section{Figure 4}

Biomarker analysis of subfractions of Fraction-I from pregnant women. Duplicate serum samples from two pregnant women (GI and G2) from the control group were separated on HPLC, and Fr-I (0-5.5 minutes) was collected in ten subfractions (each 0.5 minutes), indicated as sI to s 10 on the figure. The subfractions from one of the duplicate samples from each woman were pooled after HPLC separation, as a positive control of the complete Fr-I. After reconstitution, the samples were analyzed by E-Screen. Results are expressed as the relative proliferative effect (RPE) to E2. Values shown are mean \pm SD from triplicate wells. The broken line represents the background of the experiment (the mean of negative controls $+3 \times \mathrm{SD})$. Asterisk $(*)$ indicate significant difference $(P<0.05)$ from background.

the persistent organochlorine insecticides, $o, p^{\prime}-\mathrm{DDT}$, methoxychlor and dieldrin [18-22]. The more stable metabolite of $p, p^{\prime}$-DDT, $p, p^{\prime}$-DDE, also induced cell proliferation in accordance with previous reports $[20,23] . p, p^{\prime}-$ DDE and PCBs constitute the major part of organochlorine residues found in human adipose tissue, milk or blood [7]. Depending on the position and number of chlorine substitutions, a range of endocrine disrupting properties has been reported for different classes of PCB congeners and their metabolites [24-32]. In the present study, the major congeners PCB138, PCB153 and PCB180 did not induce an estrogenic response in the E-Screen bioassay, but they weakly inhibited E2-induced cell proliferation when analyzed in combination with E2 indicating that these compounds might antagonize the activity of endogenous estrogen. Some of the effects previously ascribed to parent PCB congeners may rather be caused by more potent metabolites, e.g., hydroxylated metabolites [32]. We examined the estrogenicity of the three most abundant hydroxylated metabolites of PCBs in human serum, and 4-OH-CB187 was found to be weakly estrogenic at the highest concentration tested. The environmental pollutant $\beta$-HCH was also found to be a potent xenoestrogen, thus confirming earlier reports [33]. Additionally, the observed responses when cells were treated with $\beta-\mathrm{HCH}, p, p^{\prime}$-DDE or methoxychlor in combination with E2, were larger than the response of E2 alone, thereby indicating that these xenoestrogenic compounds act together with endogenous estrogen to produce an enhanced estrogenic effect. Thus, our results indicate that mixtures of estrogenic compounds seem to cause additive effects and thus support reports of additive effects of estrogenic compounds in vitro $[34,35]$. The data available are, however, too limited to allow a prediction of the combined estrogenic potential from a conventional chemical concentration profile of a serum sample.

\section{Establishment of the biomarker assay}

The HPLC method was optimized to allow extraction, separation and collection of mixtures of compounds in serum samples based on their polarity. The SPE columns used for the extraction in this study constitute a "universal" polymeric sorbent that is unique due to its balanced ratio of two monomers, the lipophilic divinylbenzene, and the hydrophilic $\mathrm{N}$-vinylpyrrolidone, and allows extraction of acidic, basic, and neutral compounds in a wide range of polarities on a single SPE column. Furthermore, this mixed type of sorbent has a larger surface area and a greater capacity for a wide range of compounds than do silica-based materials [5]. 

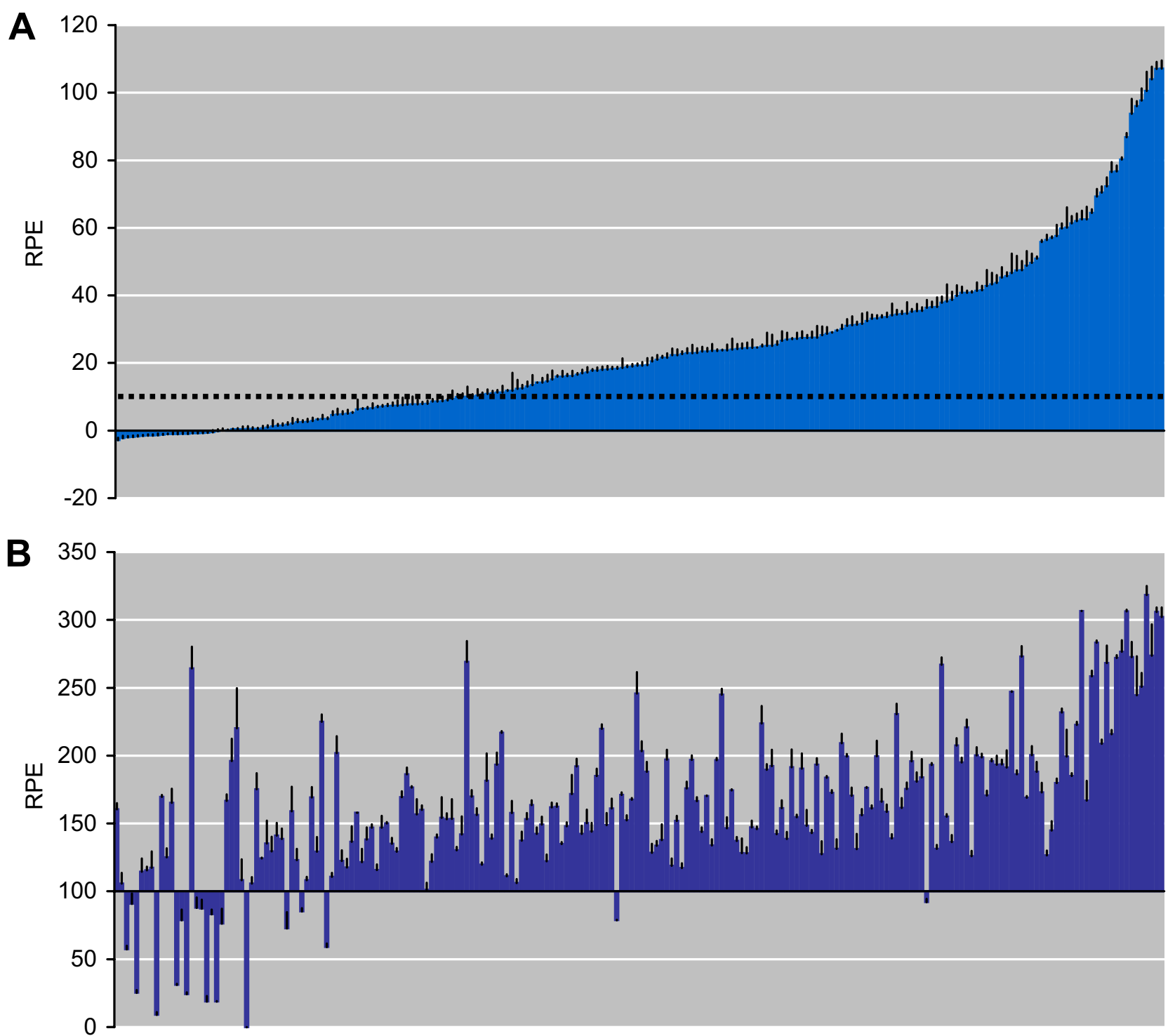

Samples $(n=211)$

\section{Figure 5}

Biomarker analysis of Fraction-I from PCB-exposed pregnant women. Serum samples $(n=2 \mathrm{II})$ from the Faroese cohort were separated on HPLC, followed by E-Screen analysis of the reconstituted $\mathrm{Fr}-\mathrm{I}$ in the absence (A) or presence (B) of 10 pM E2. Subfractions $s 4$ and $s 8$ was removed before E-Screen analysis. Results are expressed as the relative proliferative effect (RPE) to E2. Values shown are mean \pm SD from triplicate wells. The broken line represents the background of the experimental series (the mean of negative controls $+3 \times$ SD).

Our HPLC gradient system differs from that proposed by Sonnenschein, Soto and coworkers [1], because we implement a relatively long isocratic elution period prior to applying the gradient. By doing so, the lipophilic hormone-free elution period is condensed, and the elution period for midpolar compounds is expanded. This approach was chosen to increase the ability to separate compounds in the mid-polar region where endogenous hormones elute, thus allowing us to eliminate the endogenous hormones without loosing too many of the exogenous compounds. 
A caveat that needs to be addressed is that the exclusion of specific fractions, equivalent to a certain range of polarities, will potentially also exclude some exogenous compounds with retention times (polarities) identical to the endogenous hormones. This is, of course, mainly a problem when separating unknown mixtures, as individual retention times of known compounds would otherwise be available. The wide range of compounds included in this study with individual retention times demonstrates the potential of this semi-preparative method. Thus, this method will allow the user to collect fractions for specific compounds, mixtures of compounds with known polarity, or to a total serum sample after exclusion of endogenous hormones. The HPLC separation may in principle be followed by a range of different effect monitoring assays depending on the compounds separated and the focus of the research. Our focus in this study was mixtures of exogenous compounds potentially acting as xenoestrogens.

The ability to separate, collect and analyze mixtures of exogenous compounds instead of individual chemicals is a significant feature allowing evaluation of combined effects of chemicals present below their individual noobserved-effect concentrations. To test estrogenic as well as anti-estrogenic effects of mixtures of chemicals in human serum, our improved in vitro E-Screen bioassay [6] is an effect monitoring system well suited to HPLC-separated extracts. Together, the semi-preparative separation and the E-Screen bioassay act as a biomarker that will allow us not only to test individual compounds, but also to address the problems related to environmental or occupational exposure to mixtures of xenoestrogens each present at low concentrations [35].

We have used this biomarker assay to study the combined functional estrogenic response in serum from pregnant women exposed to mixtures of PCBs and other persistent organochlorines. These compounds are lipophilic to an extent that allowed us to apply the HPLC-fraction eluting within the initial 5.5 minutes (after exclusion of two minor subfractions) for E-Screen analysis. In addition, this group of women had a high level of endogenous estrogens due to pregnancy, thus making the elimination of the endogenous hormones without loosing too many of the exogenous chemicals a challenging task.

\section{Evaluation of Fraction-I from PCB-exposed women}

The biomarker analysis showed large variations in serum estrogenicity within the cohort of PCB-exposed Faroese women and more than two samples out of three induced a positive estrogenic response. In the presence of E2, an increased induction of cell proliferation compared to that of E2 alone was observed in most of these samples, thus indicating additive mixture effects.
Although it was not a main objective of this study to search for individually causative chemicals, efforts were made to assess relationships between the biomarker data and the concentrations of major environmental pollutants present in milk fat from the same subjects. Except for $\beta$-HCH, we did not find any significant correlations between the functional estrogenic response and total PCB, PCB105, PCB118, PCB138, PCB153, РCB156 or PCB180, or other environmental pollutants such as $o, p^{\prime}$-DDT, $p, p^{\prime}$ DDE, HCB, or trans-nonachlor. These chemicals were analyzed as part of a routine battery of compounds that were selected for other reasons than endocrine disruption. However, because concentrations of persistent lipophilic compounds show collinearity, these pesticide residues and persistent industrial chemicals are likely to represent the overall body burden of persistent organohalogen compounds.

A likely explanation for the lack of significant correlation between concentrations of these chemicals and our biomarker results could be that the chemical analysis did not include the chemicals primarily responsible for the biomarker effect. In addition, the concentration of the individual compounds in human serum may be below the concentration that is needed to elicit effects in our in vitro cell proliferation assay. The combined response of the mixture present in serum might, however, be significantly enhanced, even when each single compound is present below its detectable effect limit. Kortenkamp and coworkers have shown that the combined effect of a mixture of xenoestrogens can produce a significant estrogenic response even when each individual xenoestrogen is present at a concentration that alone does not produce a detectable effect $[34,35]$. As a result, an explanation in terms of single causative compounds may not be expected, because humans are exposed to complex mixtures of environmental pollutants. A number of compounds, each present at low, perhaps ineffective concentrations, may act together to produce effects that are statistically significant in the biomarker assay. Thus, the lack of correlation speaks in favor of the biomarker assay, rather than against, as the method allows detection of the combined and functional xenoestrogenic response.

\section{Conclusions}

This biomarker assay is recommended as an appropriate and useful tool for assessing the functional estrogenic potential of complex mixtures present in human serum. Because of the limited insight into the endocrine disrupting effects of single chemicals and their mixtures, it may provide a more accurate prediction of the endocrine disrupting impact than even the most detailed chemical analysis. Thus, even if the estrogenic activity of all compounds were known, the combined effect of possible additive and/or inhibiting interaction of xenoestrogens in a 
biological sample may be difficult to predict from chemical analysis of single compounds alone.

Our finding of an increased average estrogenicity of serum extracts obtained from an exposed population merits attention and should inspire further studies into the causes of this response. Our results do not necessarily indicate an increased risk of adverse effects, but it should lead to further efforts to characterize this exposure and its possible effects. For such studies, this biomarker approach may serve as a useful supplement to standard exposure assessment.

\section{List of abbreviations used}

4-OH-CB, hydroxylated metabolites of polychlorinated biphenyls; $\beta-\mathrm{HCH}, \quad \beta$-hexachlorocyclohexane; CT-FCS, charcoal/dextran-treated fetal calf serum; DMEM, Dulbecco's modified Eagle's medium; DMSO, dimethylsulfoxide; E2, 17ß-estradiol; FCS, fetal calf serum; GC, gas chromatography; HCB, hexachlorobenzene; HEPES, 4-(2hydroxyethyl)-1-piperazineethanesulfonic acid; HPLC, high-performance liquid chromatography; ICI, ICI 182,780 ; MOEC, maximum observed effect concentration; NPC, normal phase chromatography; $o, p$-DDT, $o, p^{\prime}-$ dichlorodiphenyltrichloroethane; $\mathrm{PCB}$, polychlorinated biphenyl; PE, proliferative effect; $p, p$-DDE, $p, p^{\prime}$-dichlordiphenyldichlorethylen; RPE, relative proliferative effect; $\mathrm{RPP}$, relative proliferative potency; SPE, solid-phase extraction; SRB, sulforhodamine $\mathrm{B}$; TCA, trichloroacetic acid; Tris base, 2-amino-2-(hydroxymethyl)-1,3-propanediol.

\section{Competing interests}

None declared.

\section{Authors' contributions}

THR, HRA, JBN, PW and PG participated in the design and coordination of the study. THR and FN performed the experimental work. THR drafted the manuscript. All authors read and approved the final manuscript.

\section{Additional material}

\section{Additional File 1}

Retention time and estrogenic response of analyzed compounds. A comprehensive table of the compounds (including alkyl phenols, phthalates, PCBs, organochlorine and carbamate pesticides, oral contraceptives, and endogenous estrogens) that were used in this study to characterize the biomarker assay.

Click here for file

[http://www.biomedcentral.com/content/supplementary/1476069X-2-12-S1.doc]

\section{Acknowledgments}

We thank Ana Soto for providing MCF-7 BUS cells, Aake Bergman for hydroxylated PCBs, Astra Zeneca for ICl 182,780, Birger Heinzow for unpublished data on milk lipid concentrations, Ranja Bjerring and Vibeke K. Pedersen for technical assistance. We are grateful to the cohort families for their loyal support and to the competent staff at the Faroese Hospital System.

The study received its main financial support from the Danish Environmental Research Programme via the Danish Centre for Environmental Estrogen Research. Additional support was obtained from the Danish Health Research Council, from the National Institute of Environmental Health Sciences (ES I I 68I) and from the Danish Environmental Protection Agency as part of the environmental support program, Dancea - Danish Cooperation for Environment in the Arctic. The authors are solely responsible for all results and conclusions presented in this article, and do not necessarily reflect the position of the Danish Environmental Protection Agency.

\section{References}

I. Sonnenschein C, Soto AM, Fernandez MF, Olea N, Olea-Serrano MF and Ruiz-Lopez MD: Development of a marker of estrogenic exposure in human serum. Clin Chem 1995, 41:1888-1895.

2. Soto AM, Fernandez MF, Luizzi MF, Oles Karasko AS and Sonnenschein C: Developing a marker of exposure to xenoestrogen mixtures in human serum. Environ Health Perspect 1997, 105 Suppl 3:647-654.

3. Nielsen JB, Nielsen F, Andersen HR and Grandjean P: A possible biomarker of exposure to xeno-estrogens. Epidemiology 1998, 9 Suppl 4 :S54-S54.

4. Rivas A, Fernandez MF, Cerrillo I, Ibarluzea J, Olea-Serrano MF, Pedraza $V$ and Olea N: Human exposure to endocrine disrupters: standardisation of a marker of estrogenic exposure in adipose tissue. APMIS 200I, 109: I85-197.

5. Bouvier ESP, Martin DM, Iraneta PC, Capparella M, Cheng YF and Philips DJ: A novel polymeric reversed-phase sorbent for solid phase extraction. LCGC International 1997, I0(9):577-585.

6. Rasmussen TH and Nielsen JB: Critical parameters in the MCF7 cell proliferation bioassay (E-Screen). Biomarkers 2002, 7:322-336.

7. Grandjean P, Weihe P, Needham LL, Burse VW, Patterson D.G.,Jr., Sampson EJ, Jorgensen PJ and Vahter M: Relation of a seafood diet to mercury, selenium, arsenic, and polychlorinated biphenyl and other organochlorine concentrations in human milk. Environ Res 1995, 71:29-38.

8. Fangstrom B, Athanasiadou M, Grandjean P, Weihe P and Bergman A: Hydroxylated PCB metabolites and PCBs in serum from pregnant Faroese women. Environ Health Perspect 2002, I I 0:895-899.

9. Stellman SD, Djordjevic MV, Muscat JE, Gong L, Bernstein D, Citron ML, White A, Kemeny M, Busch E and Nafziger AN: Relative abundance of organochlorine pesticides and polychlorinated biphenyls in adipose tissue and serum of women in Long Island, New York. Cancer Epidemiol Biomarkers Prev 1998, 7:489-496.

10. Sjodin A, Hagmar L, Klasson-Wehler E, Bjork J and Bergman A: Influence of the consumption of fatty Baltic Sea fish on plasma levels of halogenated environmental contaminants in Latvian and Swedish men. Environ Health Perspect 2000, 108: 1035-1041.

II. Smeds $A$ and Saukko P: Identification and quantification of polychlorinated biphenyls and some endocrine disrupting pesticides in human adipose tissue from Finland. Chemosphere 200 I, 44: I463-I47I.

12. Hovander L, Malmberg T, Athanasiadou M, Athanassiadis I, Rahm S, Bergman $A$ and Wehler EK: Identification of hydroxylated PCB metabolites and other phenolic halogenated pollutants in human blood plasma. Arch Environ Contam Toxicol 2002, 42: $105-117$

13. Rogan WJ and Ragan NB: Chemical contaminants, pharmacokinetics, and the lactating mother. Environ Health Perspect 1994, I02 Suppl I I:89-95. 
14. Bergman A, Klasson-Wehler E and Kuroki H: Selective retention of hydroxylated PCB metabolites in blood. Environ Health Perspect 1994, 102:464-469.

I5. Steuerwald U, Weihe P, Jorgensen PJ, Bjerve K, Brock J, Heinzow B, Budtz-Jorgensen E and Grandjean P: Maternal seafood diet, methylmercury exposure, and neonatal neurologic function. J Pediatr 2000, 136:599-605.

16. Brock JW, Burse VW, Ashley DL, Najam AR, Green VE, Korver MP, Powell MK, Hodge CC and Needham LL: An improved analysis for chlorinated pesticides and polychlorinated biphenyls (PCBs) in human and bovine sera using solid-phase extraction. J Anal Toxicol 1996, 20:528-536.

17. Vihma V, Adlercreutz H, Tiitinen A, Kiuru P, Wahala $\mathrm{K}$ and Tikkanen MJ: Quantitative determination of estradiol fatty acid esters in human pregnancy serum and ovarian follicular fluid. Clin Chem 2001, 47: I256-1262.

18. Soto AM, Chung KL and Sonnenschein C: The pesticides endosulfan, toxaphene, and dieldrin have estrogenic effects on human estrogen-sensitive cells. Environ Health Perspect 1994, 1 02:380-383.

19. Soto AM, Sonnenschein C, Chung KL, Fernandez MF, Olea N and Serrano FO: The E-SCREEN assay as a tool to identify estrogens: an update on estrogenic environmental pollutants. Environ Health Perspect 1995, 103 Suppl 7: I13-122.

20. Dees C, Askari M, Foster JS, Ahamed S and Wimalasena J: DDT mimicks estradiol stimulation of breast cancer cells to enter the cell cycle. Mol Carcinog 1997, I 8: 107- I I4.

21. Wade MG, Desaulniers D, Leingartner K and Foster WG: Interactions between endosulfan and dieldrin on estrogen-mediated processes in vitro and in vivo. Reprod Toxicol 1997, I I:791-798.

22. Legler J, van den Brink CE, Brouwer A, Murk AJ, van der Saag PT, Vethaak AD and van der Burg B.: Development of a stably transfected estrogen receptor-mediated luciferase reporter gene assay in the human T47D breast cancer cell line. Toxicol Sci 1999, 48:55-66.

23. Andersen HR, Andersson AM, Arnold SF, Autrup H, Barfoed M, Beresford NA, Bjerregaard P, Christiansen LB, Gissel B, Hummel R, Jorgensen EB, Korsgaard B, Le Guevel R, Leffers H, McLachlan J, Moller A, Nielsen JB, Olea N, Oles-Karasko A, Pakdel F, Pedersen KL, Perez P, Skakkeboek NE, Sonnenschein C, Soto AM and .: Comparison of short-term estrogenicity tests for identification of hormone-disrupting chemicals. Environ Health Perspect 1999, 107 Suppl I:89-108.

24. Bitman J and Cecil HC: Estrogenic activity of DDT analogs and polychlorinated biphenyls. J Agric Food Chem 1970, I 8: I I08-I I I 2.

25. Jansen HT, Cooke PS, Porcelli J, Liu TC and Hansen LG: Estrogenic and antiestrogenic actions of PCBs in the female rat: in vitro and in vivo studies. Reprod Toxicol 1993, 7:237-248.

26. Safe SH: Polychlorinated biphenyls (PCBs): environmental impact, biochemical and toxic responses, and implications for risk assessment. Crit Rev Toxicol 1994, 24:87-I49.

27. Nesaretnam K, Corcoran D, Dils RR and Darbre P: 3,4,3',4'-Tetrachlorobiphenyl acts as an estrogen in vitro and in vivo. Mol Endocrinol 1996, 10:923-936.

28. Nesaretnam $K$ and Darbre P: 3,5,3',5'-tetrachlorobiphenyl is a weak oestrogen agonist in vitro and in vivo. J Steroid Biochem Mol Biol 1997, 62:409-4l8.

29. Kramer VJ, Helferich WG, Bergman A, Klasson-Wehler E and Giesy JP: Hydroxylated polychlorinated biphenyl metabolites are anti-estrogenic in a stably transfected human breast adenocarcinoma (MCF7) cell line. Toxicol Appl Pharmacol 1997, I 44:363-376.

30. Ramamoorthy K, Vyhlidal C, Wang F, Chen I, Safe S, McDonnell DP, Leonard LS and Gaido KW: Additive estrogenic activities of a binary mixture of 2',4',6'-trichloro- and 2',3',4',5'-tetrachloro-4-biphenylol. Toxicol Appl Pharmacol 1997, 147:93-100.

31. Brouwer A, Longnecker MP, Birnbaum LS, Cogliano J, Kostyniak P, Moore J, Schantz S and Winneke G: Characterization of potential endocrine-related health effects at low-dose levels of exposure to PCBs. Environ Health Perspect 1999, I 07 Suppl 4:639-649.

32. Layton AC, Sanseverino J, Gregory BW, Easter JP, Sayler GS and Schultz TW: In vitro estrogen receptor binding of PCBs: measured activity and detection of hydroxylated metabolites in a recombinant yeast assay. Toxicol Appl Pharmacol 2002, I 80:157-163.
33. Steinmetz R, Young PC, Caperell-Grant A, Gize EA, Madhukar BV, Ben Jonathan $N$ and Bigsby RM: Novel estrogenic action of the pesticide residue beta-hexachlorocyclohexane in human breast cancer cells. Cancer Res 1996, 56:5403-5409.

34. Silva E, Rajapakse $N$ and Kortenkamp A: Something from "nothing"--eight weak estrogenic chemicals combined at concentrations below NOECs produce significant mixture effects. Environ Sci Technol 2002, 36: I75 I-I756.

35. Rajapakse N, Silva E and Kortenkamp A: Combining xenoestrogens at levels below individual no-observed-effect concentrations dramatically enhances steroid hormone action. Environ Health Perspect 2002, I 1 0:917-921.

\section{Pre-publication history}

The pre-publication history for this paper can be accessed here:

http://www.biomedcentral.com/content/backmatter/ 1476-069X-2-12-b1.pdf
Publish with Bio Med Central and every scientist can read your work free of charge

"BioMed Central will be the most significant development for disseminating the results of biomedical research in our lifetime. "

Sir Paul Nurse, Cancer Research UK

Your research papers will be:

- available free of charge to the entire biomedical community

- peer reviewed and published immediately upon acceptance

- cited in PubMed and archived on PubMed Central

- yours - you keep the copyright

Submit your manuscript here:

http://www.biomedcentral.com/info/publishing_adv.asp
BioMedcentral 